\title{
Effects of resveratrol on learning and memory in rats with vascular dementia
}

\author{
YEQING ZHANG $^{1^{*}},{\text { YUWANG } \mathrm{LI}^{2^{*}}, \text { YINXIAO WANG }}^{3}$, GENGYIN WANG $^{4}$, \\ LINGQUN MAO $^{1}$, DANHONG ZHANG ${ }^{1}$ and JINHUA WANG ${ }^{1}$

\footnotetext{
${ }^{1}$ Department of Neurology, Taizhou Central Hospital (Taizhou University Hospital), Taizhou, Zhejiang 318000;

${ }^{2}$ Department of Neurology, Tianjin Huanhu Hospital, Tianjin 300060; ${ }^{3}$ School of Public Health,
} \\ ${ }^{4}$ School of Basic Medicine, North China University of Science and Technology, Tangshan, Hebei 063210, P.R. China
}

Received January 26, 2019; Accepted July 19, 2019

DOI: $10.3892 / \mathrm{mmr} .2019 .10723$

\begin{abstract}
The purpose of the present study was to study the effects of resveratrol on cognitive function in rats with vascular dementia and to investigate the molecular mechanisms of its neuroprotective effects. Forty-five SD rats were randomly divided into 3 groups: The control group (Con group, $n=15$ ), the model group (VD group, $n=15$ ) and the resveratrol-treated VD group (Res group, $n=15$ ). The VD rats (the VD group and the Res group) were generated by bilateral common carotid artery occlusion. The rats in the Res group received daily resveratrol treatment intraperitoneally for 4 weeks. Cognitive function was tested using the Morris water maze test. The levels of SOD and MDA (oxidative stress indicators) were detected by ELISA kits. The protein expression of Bax, Bcl-2 and caspase-3 was detected by western blotting. Compared with the rats in the Con group, the rats in the VD group exhibited decreased cognitive function, significantly increased hippocampal content of MDA, Bax and caspase-3 $(\mathrm{P}<0.05)$, and significantly reduced hippocampal expression of SOD and Bcl-2 $(\mathrm{P}<0.05)$. Compared with the rats in the VD group, the rats in the Res group exhibited increased cognitive ability, reduced hippocampal content of MDA, Bax and caspase-3 $(\mathrm{P}<0.05)$, and increased hippocampal expression of SOD and Bcl-2 $(\mathrm{P}<0.05)$. Resveratrol treatment significantly improved the spatial learning and memory of the VD rats. The mechanism associated with the neuroprotective effects
\end{abstract}

Correspondence to: Dr Jinhua Wang, Department of Neurology, Taizhou Central Hospital (Taizhou University Hospital), 999 Donghai Avenue, Economic Development Zone, Taizhou, Zhejiang 318000, P.R. China

E-mail:wjh19852008@sina.com

*Contributed equally

Abbreviations: VD, vascular dementia; BCCAO, bilateral common carotid artery occlusion; MWM, Morris water maze

Key words: resveratrol, VD, cognition, oxidative stress, apoptosis of resveratrol may be closely related to the inhibition of the apoptosis pathway and oxidative stress injury.

\section{Introduction}

Vascular dementia (VD) is the second most common type of dementia following Alzheimer's disease and is characterized by a gradual decline in learning and memory ability $(1,2)$. VD may affect more individuals in the future as the population ages and survival after cardiovascular disease and stroke increases (3). The diagnosis of VD includes a decline in cognitive abilities, resulting in impaired functional abilities. To date, there is no effective treatment for VD, and the exact mechanisms of VD remain unclear. Therefore, it is important to study the pathogenesis and treatment of VD.

Resveratrol is a natural phenolic substance found in the epidermis of red grapes and red wine, and it is abundant in Polygonum cuspidatum (4). Numerous studies have shown that resveratrol has many biological properties (5-7), including anti-oxidative, antitumor and anti-inflammatory properties. Resveratrol has been shown to improve the histopathological and behavioral outcomes of various types of acute central nerve injury, including stroke, traumatic brain injury, spinal cord injury and subarachnoid hemorrhage (8-11). Resveratrol also exhibits neuroprotective effects in Alzheimer's disease $(12,13)$, showing cognitive-enhancing effects. However, there is a lack of data concerning the role of resveratrol in VD. The present study used bilateral common carotid artery occlusion (BCCAO) to establish a rat model of VD, and the effects of resveratrol on cognitive function in rats with VD and the related mechanisms of action of resveratrol were explored by detecting the change in cognitive function of the rats using a water maze test.

\section{Materials and methods}

Animals. Forty-five healthy male Sprague-Dawley rats aged 2 months and weighing 200-230 g were obtained from the Animal Center of Hebei Medical University. The animals were housed in a room with a constant temperature of $23 \pm 2^{\circ} \mathrm{C}$ on a 12-h light-dark cycle (lights on at 8:00 a.m.). All rats were allowed free access to food and water. All experiments were 
carried out in accordance with the regulations of the Ethics Committee of Taizhou Central Hospital (Taizhou University Hospital, Taizhou, Zhejiang).

Bilateral common carotid artery occlusion (BCCAO). The VD rats (the VD group and the Res group) were generated by bilateral common carotid artery occlusion. The rats were fasted $12 \mathrm{~h}$ before the operation, and the rats were anesthetized with chloral hydrate $(10 \%, 0.3 \mathrm{ml} / 100 \mathrm{~g})$ by intraperitoneal injection. After the rats were anesthetized, the skin was cut along the midline of the neck to expose the bilateral common carotid arteries. Then, the surrounding tissues were carefully separated. The bilateral common carotid arteries were tied with silk sutures (approximately $1 \mathrm{~cm}$ inferior to the origin of the external carotid artery). The rats in the control group were subjected to the same surgical procedure without the occlusion of the bilateral common carotid arteries. After the operation, the skin was stitched together and sterilized, and the animals were placed in cages at room temperature and allowed to wake up naturally and drink water normally.

Resveratrol administration. Forty-five SD rats were randomly divided into 3 groups: the control group (Con group, $n=15$ ), the model group (VD group, $n=15$ ) and the resveratrol-treated VD group (the Res group, $n=15$ ). We used bilateral common carotid artery occlusion to establish VD in the rats (the VD group and the Res group). The rats in the Res group received daily treatment with resveratrol (Sigma-Aldrich; Merck KGaA; 20 and $10 \mathrm{ml} / \mathrm{kg}$ ) intraperitoneally for 4 weeks. The rats in the other groups received saline intraperitoneally.

Morris water maze (MWM) test. Following 4 weeks of resveratrol treatment, the cognitive ability of the rats was tested by MWM, which measures spatial learning and memory ability. The animals were subjected to a daily session of four training trials for 5 consecutive days. In each training session, the rats were placed into the pool at four different starting points (different quadrants). The experimental scheme is shown in Table I. The rats were then permitted to find the platform within a maximum time period of $120 \mathrm{sec}$, and the rats were allowed to remain on the platform for an additional $20 \mathrm{sec}$. The time each rat took to find the platform was recorded as the escape latency. If a rat was unable to find the platform within the 120 -sec time period, the rat was then guided onto the platform by the experimenter and kept there for $20 \mathrm{sec}$, and the escape latency was recorded as $120 \mathrm{sec}$. On day 6 of the probe test, the platform was removed, and the rats were placed into water and allowed to swim freely to find the removed platform for 120 sed. The activity of each rat was recorded by a video tracking system. The escape latency and the number of times the platform location was crossed (crossing frequency) were recorded.

Superoxide dismutase (SOD) and malondialdehyde (MDA) measurements. Following the MWM test, 5 rats from each group were randomly selected, anaesthetized (sodium pentobarbital; 60 mg/kg; Sinopharm Chemical Reagent Co., Ltd.), and immediately sacrificed by cervical location, and the brain tissues were quickly isolated. The hippocampus was separated for further use. The expression of SOD was detected using the method described by Winterbourn et al (14). For the assay, $0.067 \mathrm{M}$ potassium phosphate buffer ( $\mathrm{pH}$ 7.8) was added to $0.1 \mathrm{M}$ EDTA containing $0.3 \mathrm{mM}$ sodium cyanide, $1.5 \mathrm{mM}$ NBT and $0.1 \mathrm{ml}$ of the sample. Then, $0.12 \mathrm{mM}$ riboflavin was added to each sample to initiate the reaction, and the samples were incubated for $12 \mathrm{~min}$. The absorbance of the sample was read at $560 \mathrm{~nm}$ on a Genesys $10 \mathrm{UV}$ (Thermo Fisher Scientific, Inc.) spectrophotometer for $5 \mathrm{~min}$. The amount of enzyme required to produce $50 \%$ inhibition was $1 \mathrm{U}$, and the results are expressed as U/mg protein (14). MDA was detected using the method described by Riahi et al (15). The homogenized solution $(0.1 \mathrm{ml})$ was deproteinized by the addition of $0.2 \mathrm{ml}$ of a zinc sulfate solution and then centrifuged at 4,000 x g and $4^{\circ} \mathrm{C}$ for $20 \mathrm{~min}$ to separate the supernatant for nitrate determination. The supernatant of each sample $(0.1 \mathrm{ml})$, pure water (as a blank) or sodium nitrite (as a standard) was mixed with $0.1 \mathrm{ml}$ of vanadium chloride III to reduce the nitrite to nitrate. Then, $0.05 \mathrm{ml}$ of sulfonamide $(0.01 \%)$ and $0.05 \mathrm{ml}$ of $\mathrm{N}$-[1-naphthyl] ethylenediamine dihydrochloride (NED, $0.01 \%$ ) were incubated for $30 \mathrm{~min}$ at $37^{\circ} \mathrm{C}$ in the dark. Thereafter, the absorbance of the solution was measured at a wavelength of $540 \mathrm{~nm}$. The nitrate concentration was estimated from the standard curve produced by the absorbance of each sodium nitrate solution. The nitrate level is expressed as $\mathrm{mmol} / \mathrm{mg}$ protein. The antibodies were as follows: SOD (CAS: A001-1-1, Nanjing Jiancheng Bioengineering Institute, China) and MDA (CAS: A003-1-1, Nanjing Jiancheng Bioengineering Institute, China).

TUNEL assay. Apoptosis of hippocampal neurons was detected by a TUNEL assay. The brain tissues were fixed in 4\% PFA and embedded in paraffin wax. An In Situ Cell Death Detection Kit (Roche, Switzerland) was used to detect apoptosis according to the manufacturer's instructions. Apoptotic changes were measured via fluorescence microscopy (Olympus, Tokyo, Japan). The images were captured using a light microscope (magnification, $\mathrm{x} 400$; Olympus Corporation). The apoptosis rate was analyzed as described in a previous study (16).

Western blot analysis. The protein expression of Bax, Bcl-2 and caspase- 3 was detected by western blot analysis. Five rats from each group were randomly selected and anaesthetized, and the brain tissues were quickly isolated. The hippocampus was rapidly separated on ice and then quickly frozen and stored at $-80^{\circ} \mathrm{C}$ for further use. The hippocampus was homogenized with radioimmunoprecipitation assay lysis buffer. The proteins were then separated by $10 \%$ SDS-PAGE and transferred onto PVDF membranes. At room temperature, the membranes were blocked with $5 \%$ skim milk for $2 \mathrm{~h}$ and then incubated with primary antibodies overnight at $4^{\circ} \mathrm{C}$. The antibodies were as follows: caspase-3 (1:2,000, Abcam, ab13847), Bcl-2 (1:1,000, ab196495, Abcam), Bax (1:2,000, Abcam, ab32503), and $\beta$-actin (1:2,000, Abcam, ab179467). At room temperature, the membranes were incubated with horseradish peroxidase-conjugated secondary antibodies (1:3,000 diluted, Abcam, ab7097) for $1 \mathrm{~h}$ after several washes. We detected the proteins using a chemiluminescence system. Quantity One software (https://download.csdn.net/download/ sinat_29367613/8846211) v4.62 was used to quantify the 
Table I. The experimental scheme for the Morris water maze (MWM) test.

\begin{tabular}{lllll}
\hline Day & Trial 1 & Trial 2 & Trial 3 & Trial 4 \\
\hline 1 & $\mathrm{~N}$ & $\mathrm{E}$ & $\mathrm{SE}$ & $\mathrm{NW}$ \\
2 & $\mathrm{SE}$ & $\mathrm{N}$ & $\mathrm{NW}$ & $\mathrm{E}$ \\
3 & $\mathrm{NW}$ & $\mathrm{SE}$ & $\mathrm{E}$ & $\mathrm{N}$ \\
4 & $\mathrm{E}$ & $\mathrm{NW}$ & $\mathrm{N}$ & $\mathrm{SE}$ \\
5 & $\mathrm{~N}$ & $\mathrm{SE}$ & $\mathrm{E}$ & $\mathrm{NW}$ \\
6 (Probe) & $\mathrm{NE}$ & & & \\
\hline
\end{tabular}

N, North; E, East; SE, Southeast; NW, Northwest.

protein bands. All relative optical densities were normalized to $\beta$-actin.

Statistical analysis. Statistical analysis was performed using SPSS 17.0 software (SPSS, Inc., Chicago, IL, USA). All data are presented as the mean \pm SD. Differences among three or more groups were compared by one-way analysis of variance (ANOVA) followed by Bonferroni tests to explain the exact difference between the means. P-values of 0.05 or less were regarded as indicative of a statistically significant difference.

\section{Results}

Resveratrol improves cognitive function in VD rats. We used the MWM test to evaluate the learning and memory ability of the different groups to determine whether resveratrol improves cognitive function in VD rats (Fig. 1). Compared to that in the control group, the escape latency in the VD group was significantly increased. However, after 4 weeks of resveratrol administration, the escape latency was significantly decreased (Fig. 1A). In the probe test, compared to that in the control group, the crossing frequency in the VD group was significantly decreased. Compared to that in the VD group, the crossing frequency in the Res group was significantly increased (Fig. 1B).

Resveratrol reduces the concentration of MDA and increases the concentration of SOD in VD rats. Compared with that in the Con group, the hippocampal content of MDA in the VD group was significantly increased, and the hippocampal expression of SOD was significantly reduced. Compared with that in the VD group, the hippocampal content of MDA in the Res group was significantly reduced, and the expression of hippocampal SOD was significantly increased. The results indicated that the ability of the rats in the VD group to recover from oxidative stress was impaired and that the ability of the VD rats to recover from oxidative stress was improved after resveratrol treatment but did not reach the level of the Con group. The hippocampal expression of SOD and MDA is shown in Fig. 2.

Resveratrol prevents the apoptosis of hippocampal neurons in the CAl region. The data were produced using TUNEL assay. As shown in Fig. 3, the number of apoptotic neurons in the
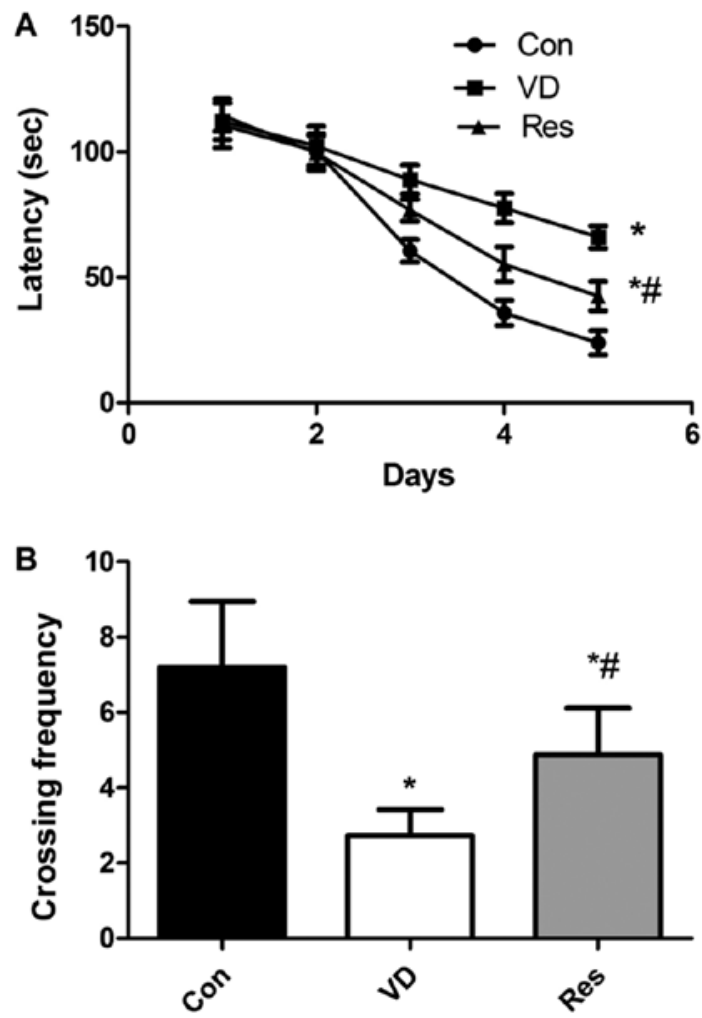

Figure 1. Results of the MWM test. (A) Compared to that in the control group, the escape latency (in sec) in the VD group was significantly increased. However, after 4 weeks of resveratrol administration, the escape latency was significantly decreased. (B) Compared to that in the control group, the crossing frequency in the VD group was significantly decreased. Compared to that in the VD group, the crossing frequency in the Res group was significantly increased. ${ }^{*} \mathrm{P}<0.05$ vs. the Con group; ${ }^{\prime} \mathrm{P}<0.05$ vs. the VD group. MWM, Morris water maze; VD, vascular dementia; Con, control; Res, resveratrol

CA1 region of the model group was significantly increased. However, resveratrol significantly reduced the number of apoptotic neurons in the CA1 region.

Resveratrol decreases hippocampal apoptosis in VD rats. The western blot analysis results (Fig. 4), showed that compared with that in the Con group, the expression of Bcl-2 in the VD group was significantly decreased. Compared with that in the VD group, the expression of Bcl-2 in the Res group was significantly increased. Compared with that in the Con group, the expression of hippocampal Bax and caspase-3 in the VD group was increased. However, hippocampal Bax and caspase-3 protein expression was significantly decreased after 4 weeks of resveratrol treatment. These results revealed that cerebral hypoperfusion inhibited Bcl-2 expression and enhanced hippocampal Bax and caspase-3 expression. Furthermore, resveratrol treatment enhanced the expression of Bcl-2 and inhibited Bax and caspase-3 expression in the hippocampus of VD rats.

\section{Discussion}

Dementia is an acquired mental deficiency that causes severe social or occupational impairment. Vascular dementia (VD) is considered the second most common type of dementia, 


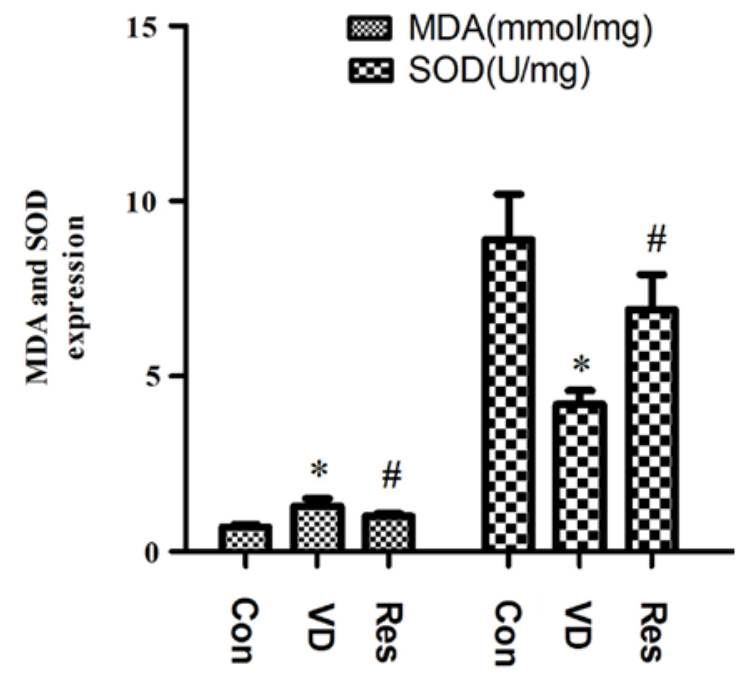

Figure 2. Results of MDA and SOD measurements. Compared with that in the Con group, the hippocampal content of MDA in the VD group was significantly increased, and the hippocampal expression of SOD was significantly reduced. However, after 4 weeks of resveratrol administration, the expression of MDA and SOD was reversed. "P $<0.05$ vs. the Con group; ${ }^{\#} \mathrm{P}<0.05$ vs. the VD group. MDA, malondialdehyde; SOD, superoxide dismutase; VD, vascular dementia; Con, control; Res, resveratrol.

including dementia resulting from all types of vascular disease (17). When the brain's blood supply is blocked or the diseased blood vessel system is impaired, VD can occur leading to a gradual decline in memory and cognitive function (18). Bilateral carotid artery occlusion (BCCAO) can lead to the chronic hypoperfusion of the brain in rats, resulting in severe leukodystrophy and learning and memory impairment (19). Therefore, BCCAO is useful for studying the mechanism of chronic cerebral hypoperfusion and searching for drugs for VD in rat models. Resveratrol is a natural phenolic substance found in the epidermis of red grapes and red wine, and it is abundant in Polygonum cuspidatum. Numerous studies have shown that resveratrol has many biological properties, including antioxidative, antitumor and anti-inflammatory properties. Although many studies have reported a close link between resveratrol and cognitive ability, the underlying mechanism remains unclear. Therefore, we investigated the effect of resveratrol on the cognitive ability of rats with VD. Our results showed that the cognitive ability of VD rats decreased and that resveratrol improved the cognitive function of VD rats. The results showed that resveratrol is a potential neuroprotective drug that can counter the cognitive impairment of BCCAO-induced VD in rat models.

The pathogenesis of VD includes oxidative stress, the cytotoxicity of reactive oxygen, mitochondrial dysfunction and apoptosis $(20,21)$. Oxidative stress is caused by changes in the body's oxidation and antioxidant balance, which is more conducive to producing free radicals or reactive oxygen free radicals rather than other antioxidant defense mechanisms (22). Oxidative stress is a regulator of neurological injury and cognitive dysfunction after stroke, and a large number of studies have found oxidative DNA damage around cerebral infarctions in rats (23-25). Oxidative stress is not only associated with VD but is also associated with all risk factors for VD. Malondialdehyde (MDA) and superoxide dismutase (SOD) are widely used in many studies to represent the level of oxidative stress $(26,27)$. MDA, a common marker for oxidative stress injury, is the product of lipid peroxidation in oxidative stress and is positively correlated with oxidative stress and lipid peroxidation (26). Patients with VD have been found to have high levels of MDA, and MDA levels in these patients are higher than in patients with Alzheimer's disease (28). Clinical and experimental studies on cerebrovascular diseases have found that the peroxidative damage of free radicals is a likely mechanism of VD. SOD is an important antioxidant enzyme in the body. It is involved in the most important antioxidant system in the body and can directly scavenge free radicals and eliminate peroxidative damage. Free radicals obviously damage learning and memory. Clinical trials have found that oxidative stress plays a role in brain damage in VD patients, which may be related to the decreased SOD activity in red blood cells of VD patients, especially in patients with cerebrovascular lesions (29). The results of the present study showed that MDA expression in VD rats was increased and that the activity of SOD in VD rats was decreased, indicating that bilateral carotid artery occlusion resulted in chronic ischemia and further led to oxidative stress injury in the VD rat model. Four weeks of resveratrol administration reversed the expression of MDA and SOD, confirming that resveratrol treatment improved the spatial learning and memory function of VD rats and may be closely related to anti-oxidative stress injury.

Apoptosis is a process of programmed cell death, and the term is derived from a Greek word meaning the falling of leaves from a tree. In many different mechanisms of cell death, cell apoptosis has been proposed to explain the cell loss observed in many neurological disorders, such as Alzheimer's disease, VD, Parkinson's disease, amyotrophic lateral sclerosis muscular atrophy, and stroke (30). Bcl-2 and $\mathrm{Bax}$ are the two main genes responsible for regulating cell apoptosis. Bcl-2 is a key member of the anti-apoptotic Bcl-2 family and is essential for regulating cell apoptosis mediated by mitochondria $(31,32)$. The overexpression of Bcl-2 has been shown to protect nerve cells from damage by neurotoxins (33). Bax, which promotes the release of cytochrome $c$ by transferring it to the mitochondrial membrane, thereby promoting downstream cell apoptosis, is a member of the survivin subfamily (34). Caspase, which is an important medium for apoptosis is a member of the cysteine protease family. Caspases mainly participate in the process of apoptosis through two different pathways: the death receptor pathway and the mitochondrial pathway (35). Caspase-3 plays the role of the apoptotic executor, regardless of the pathway involved. Caspase-3 is a type of pro-apoptotic enzyme that activates the DNA fragmentation factor and then activates endonucleases to split nuclear DNA, eventually leading to apoptosis $(36,37)$. Research has confirmed that apoptosis is an important molecular biological mechanism that is closely related to VD (38). In the present study, the protein expression of Bax, Bcl-2 and caspase-3 was detected by western blot analysis. The results showed that the hippocampal expression of Bax and caspase- 3 was significantly increased and that the expression of hippocampal Bcl-2 was significantly reduced, confirming the activation of the hippocampal apoptosis pathway in VD rats. In the Res group, the hippocampal 
A

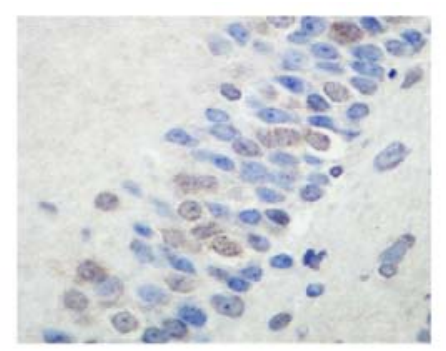

B

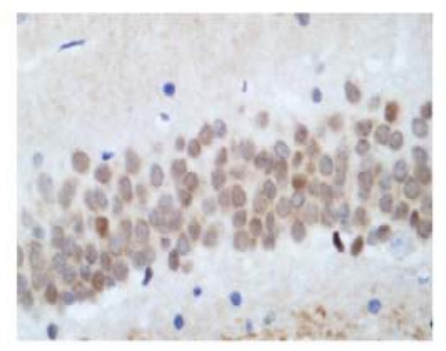

C

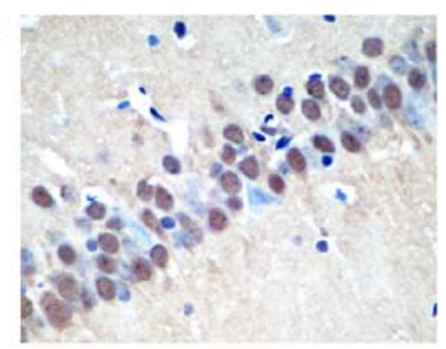

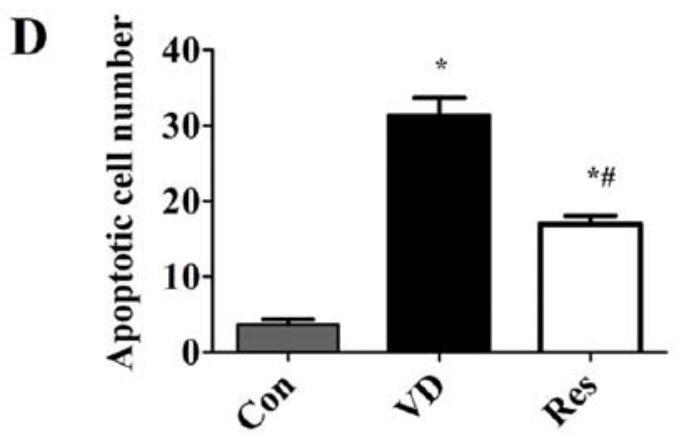

Figure 3. Resveratrol mitigates neuronal apoptosis in VD rats. (A) Fewer apoptotic neurons were found in the control group. (B) A significant increase in the number of apoptotic neurons was found in the VD model. (C) Apoptotic neurons were decreased in the Res treatment group. (D) Quantification of apoptotic cells. ${ }^{*} \mathrm{P}<0.05$ vs. the Con group; ${ }^{\#} \mathrm{P}<0.05$ vs. the VD group. VD, vascular dementia; Con, control; Res, resveratrol.

A
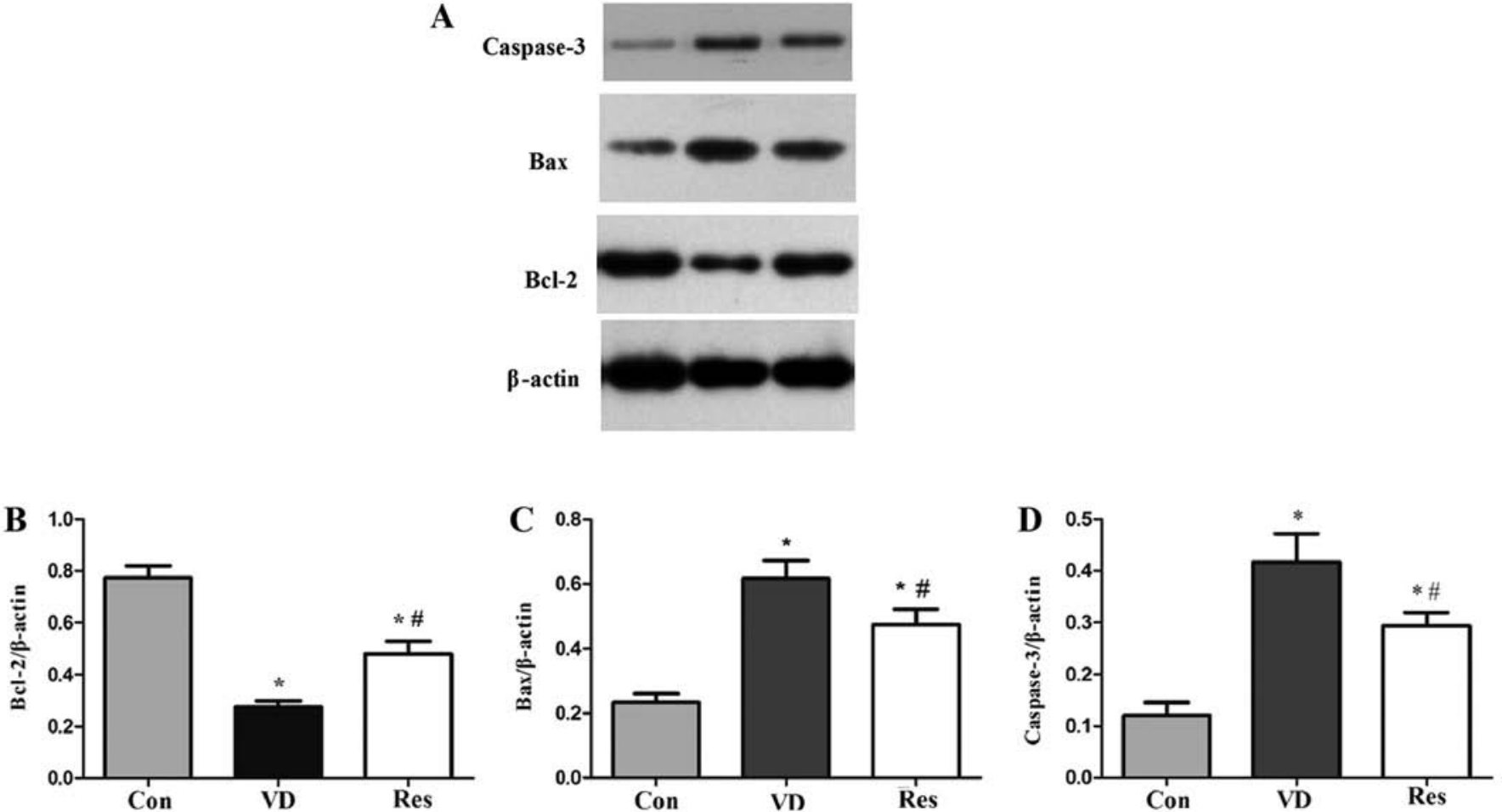

Figure 4. Western blot analysis of Bcl-2, Bax and caspase-3 expression. (A) Bcl-2, Bax, caspase- 3 and $\beta$-actin protein bands. (B) The relative protein expression of Bcl-2. Compared with that in the Con group, the expression of Bcl-2 in the VD group was significantly decreased. Compared with that in the VD group, the expression of Bcl-2 in the Res group was significantly increased. (C) The relative protein expression of Bax. Compared with that in the Con group, the expression of Bax in the VD group was significantly increased. However, hippocampal Bax protein expression was significantly decreased after 4 weeks of resveratrol treatment. (D) The relative protein expression of caspase-3. Compared with that in the Con group, the expression of caspase-3 in the VD group was significantly increased. However, hippocampal caspase-3 protein expression was significantly decreased after 4 weeks of resveratrol treatment. " $\mathrm{P}<0.05$ vs. the Con group; ${ }^{*} \mathrm{P}<0.05$ vs. the VD group. VD, vascular dementia; Con, control; Res, resveratrol; Bcl-2, B-cell lymphoma 2; Bax, Bcl-2-like protein 4.

expression of Bax and caspase- 3 was decreased, and the hippocampal expression of $\mathrm{Bcl}-2$ was increased, indicating that resveratrol has obvious anti-apoptotic properties in VD rats. 
In conclusion, resveratrol significantly improved cognitive impairment, decreased MDA levels, increased SOD activity and altered the expression of apoptotic proteins in VD rats. The mechanism of its neuroprotective effects may be closely related to the inhibition of the apoptosis pathway and oxidative stress injury. The results of this study confirmed the neuroprotective effects of resveratrol treatment in VD rats, providing a theoretical basis for the basic and clinical treatment of VD and other types of dementia. Nevertheless, our experiments found that resveratrol treatment did not improve cognitive function in VD rats completely, showing the limitations of this treatment, and the molecular mechanisms remain to be further explored in future experiments. In addition, in future research, we will design a positive control trial and determine whether resveratrol is superior to other drugs. The rats had only mild cognitive impairment, thus we used short-term intervention. If rats develop severe cognitive impairment or even dementia, the damage is irreversible, and even long-term intervention cannot reverse dementia. Of course, in future studies, we will compare whether short-term and long-term interventions have an impact on treatment outcomes. Moreover, $\beta$-amyloid and tau, markers for VD, will be tested in the future to strengthen our research findings.

\section{Acknowledgements}

Not applicable.

\section{Funding}

This study was funded by grants from the Taizhou Science and Technology Planning Project (2018, 1801ky33).

\section{Availability of data and materials}

All data generated or analyzed during this study are included in this published article.

\section{Authors' contributions}

YZ and JW made substantial contributions to the conception and design of the study. YL made substantial contributions to the conception and design of the study, and drafted and revised the manuscript. YW, LM and DZ made contributions to the acquisition of data. GW made contributions to the analysis and intepretation of data. All authors read and approved the manuscript and agree to be accountable for all aspects of the research in ensuring that the accuracy or integrity of any part of the work are appropriately investigated and resolved.

\section{Ethics approval and consent to participate}

All experiments were carried out in accordance with the regulations of the Ethics Committee of Taizhou Central Hospital (Taizhou University Hospital, Taizhou, Zhejiang).

\section{Patient consent for publication}

Not applicable.

\section{Competing interests}

The authors declare that they have no competing interests.

\section{References}

1. Roman GC: Facts, myths, and controversies in vascular dementia. J Neurol Sci 226: 49-52, 2004.

2. Rockwood K, Wentzel C, Hachinski V, Hogan DB, MacKnight C and McDowell I: Prevalence and outcomes of vascular cognitive impairment. Vascular Cognitive Impairment Investigators of the Canadian Study of Health and Aging. Neurology 54: 447-451, 2000.

3. Levine DA and Langa KM: Vascular cognitive impairment: Disease mechanisms and therapeutic implications. Neurotherapeutics 8: 361-373, 2011.

4. Villaflores OB, Chen YJ, Chen CP, Yeh JM and Wu TY: Curcuminoids and resveratrol as anti-Alzheimer agents. Taiwan J Obstet Gynecol 51: 515-525, 2012.

5. Wood LG, Wark PA and Garg ML: Antioxidant and anti-inflammatory effects of resveratrol in airway disease. Antioxid Redox Signal 13: 1535-1548, 2010.

6. Chang CC, Chang CY, Huang JP and Hung LM: Effect of resveratrol on oxidative and inflammatory stress in liver and spleen of streptozotocin-induced type 1 diabetic rats. Chin J Physiol 55: 192-201, 2012.

7. Richard T, Pawlus AD, Iglésias ML, Pedrot E, Waffo-Teguo P, Mérillon JM and Monti JP: Neuroprotective properties of resveratrol and derivatives. Ann N Y Acad Sci 1215: 103-108, 2011.

8. Simão F, Matté A, Breier AC, Kreutz F, Trindade VM, Netto CA and Salbego CG: Resveratrol prevents global cerebral ischemia-induced decrease in lipid content. Neurol Res 35: 59-64, 2013.

9. Girbovan C, Morin L and Plamondon H: Repeated resveratrol administration confers lasting protection against neuronal damage but induces dose-related alterations of behavioral impairments after global ischemia. Behav Pharmacol 23: 1-13, 2012.

10. Shao AW, Wu HJ, Chen S, Ammar AB, Zhang JM and Hong Y: Resveratrol attenuates early brain injury after subarachnoid hemorrhage through inhibition of NF-kappaB-dependent inflammatory/MMP-9 pathway. CNS Neurosci Ther 20: 182-185, 2014.

11. Ates O, Cayli S, Altinoz E, Gurses I, Yucel N, Kocak A, Yologlu $S$ and Turkoz Y: Effects of resveratrol and methylprednisolone on biochemical, neurobehavioral and histopathological recovery after experimental spinal cord injury. Acta Pharmacol Sin 27: 1317-1325, 2006.

12. Li F, Gong Q, Dong H and Shi J: Resveratrol, a neuroprotective supplement for Alzheimer's disease. Cur Des 18: 27-33, 2012.

13. Ma T, Tan MS, Yu JT and Tan L: Resveratrol as a Therapeutic Agent for Alzheimer's Disease. Biomed Res Int 2014: 350516, 2014.

14. Winterbourn CC, Peskin AV and Parsons-Mair HN: Thiol oxidase activity of copper, zinc superoxide dismutase. J Biol Chem 277: 1906-1911, 2002.

15. Riahi S, Mohammadi MT, Sobhani V and Soleimany M: Chronic effects of aerobic exercise on gene expression of LOX-1 receptor in the heart of rats fed with high fat diet. Iran J Basic Med Sci 18: 805-812, 2015 .

16. Wang G, Fang H, Zhen Y, Xu G, Tian J, Zhang Y, Zhang D, Zhang G, Xu J, Zhang Z, et al: Sulforaphane prevents neuronal apoptosis and memory impairment in diabetic rats. Cell Physiol Biochem 39: 901-907, 2016.

17. Román GC: Vascular dementia may be the most common form of dementia in the elderly. J Neurol Sci 203-204: 7-10, 2002.

18. Jellinger KA: The enigma of vascular cognitive disorder and vascular dementia. Acta Neuropathol 113: 349-388, 2007.

19. Ni J, Ohta H, Matsumoto $\mathrm{K}$ and Watanabe H: Progressive cognitive impairment following chronic cerebral hypoperfusion induced by permanent occlusion of bilateral carotid arteries in rats. Brain Res 653: 231-236, 1994.

20. Wang J, Zhang HY and Tang XC: Cholinergic deficiency involved in vascular dementia: Possible mechanism and strategy of treatment. Acta Pharmacol Sin 30: 879-888, 2009.

21. Bennett S, Grant MM and Aldred S: Oxidative stress in vascular dementia and Alzheimer's disease: A common pathology. J Alzheimers Dis 17: 245-257, 2009. 
22. Bhatti JS, Kumar S, Vijayan M, Bhatti GK and Reddy PH: Therapeutic strategies for mitochondrial dysfunction and oxidative stress in age-related metabolic disorders. Prog Mol Biol Transl Sci 146: 13-46, 2017.

23. Nagayama T, Lan J, Henshall DC, Chen D, O'Horo C, Simon RP and Chen J: Induction of oxidative DNA damage in the peri-infarct region after permanent focal cerebral ischemia. J Neurochem 75: 1716-1728, 2000.

24. Nanetti L, Taffi R, Vignini A, Moroni C, Raffaelli F, Bacchetti T, Silvestrini M, Provinciali L and Mazzanti L: Reactive oxygen species plasmatic levels in ischemic stroke. Mol Cell Biochem 303: 19-25, 2007.

25. Raz L, Zhang QG, Zhou CF, Han D, Gulati P, Yang LC, Yang F, Wang RM and Brann DW: Role of Racl GTPase in NADPH oxidase activation and cognitive impairment following cerebral ischemia in the rat. PLoS One 5: e12606, 2010.

26. Liu H, Zhao M, Yang S, Gong DR, Chen DZ and Du DY: (2R,3S)-Pinobanksin-3-cinnamate improves cognition and reduces oxidative stress in rats with vascular dementia. J Nat Med 69: 358-365, 2015.

27. Ghorbanzadeh V, Mohammadi M, Mohaddes G, Dariushnejad H, Chodari L and Mohammadi S: Protective effect of crocin and voluntary exercise against oxidative stress in the heart of high-fat diet-induced type 2 diabetic rats. Physiol Int 103: 459-468, 2016.

28. Gustaw-Rothenberg K, Kowalczuk K and Stryjecka-Zimmer M Lipids' peroxidation markers in Alzheimer's disease and vascular dementia. Geriatr Gerontol Int 10: 161-166, 2010.

29. Wallin A, Kapaki E, Boban M, Engelborghs S, Hermann DM, Huisa B, Jonsson M, Kramberger MG, Lossi L, Malojcic B, et a1: Biochemical markers in vascular cognitive impairment associated with subcortical small vesseldisease-A consensus report. BMC Neurol 17: 102, 2017.

30. Favaloro B, Allocati N, Graziano V, Di Ilio C and De Laurenzi V: Role of apoptosis in disease. Aging (Albany NY) 4: 330-349, 2012.
31. Garner TP, Lopez A, Reyna DE, Spitz AZ and Gavathiotis E: Progress in targeting the BCL-2 family of proteins. Curr Opin Chem Biol 39: 133-142, 2017.

32. Brown LM, Hanna DT, Khaw SL and Ekert PG: Dysregulation of BCL-2 family proteins by leukemia fusion genes. J Biol Chem 292: 14325-14333, 2017.

33. Gustafsson AB and Gottlieb RA: Bcl-2 family members and apoptosis, taken to heart. Am J Physiol Cell Physiol 292: C45-C51, 2007.

34. Wolter KG, Hsu YT, Smith CL, Nechushtan A, Xi XG and Youle RJ: Movement of Bax from the cytosol to mitochondria during apoptosis. J Cell Biol 139: 1281-1292, 1997.

35. Grütter MG: Caspases: Key players in programmed cell death. Curr Opin Struct Biol 10: 649-655, 2000.

36. Sun ZK, Ma XR, Jia YJ, Liu YR, Zhang JW and Zhang BA: Effects of resveratrol on apoptosis in a rat model of vascular dementia. Exp Ther Med 7: 843-848, 2014.

37. Chang CT, Korivi M, Huang HC, Thiyagarajan V, Lin KY, Huang PJ, Liu JY, Hseu YC and Yang HL: Inhibition of ROS production, autophagy or apoptosis signaling reversed the anticancer properties of Antrodia salmonea in triple-negative breast cancer (MDA-MB-231) cells. Food Chem Toxicol 103: 1-17, 2017.

38. Zhang Y, Wang LL, Wu Y, Wang N, Wang SM, Zhang B, Shi CG and Zhang SC: Paeoniflorin attenuates hippocampal damage in a rat model of vascular dementia. Exp Ther Med 12: 3729-3734, 2016.

This work is licensed under a Creative Commons Attribution-NonCommercial-NoDerivatives 4.0 International (CC BY-NC-ND 4.0) License. 